\title{
Social comparison modulates reward-driven attentional capture
}

\author{
Jun Jiao $\cdot$ Feng Du $\cdot$ Xiaosong He $\cdot$ Kan Zhang
}

Published online: 25 February 2015

(C) Psychonomic Society, Inc. 2015

\begin{abstract}
It is well established that attention can be captured by task irrelevant and non-salient objects associated with value through reward learning. However, it is unknown whether social comparison influences reward-driven attentional capture. The present study created four social contexts to examine whether different social comparisons modulate the rewarddriven capture of attention. The results showed that rewarddriven attentional capture varied with different social comparison conditions. Most prominently, reward-driven attentional capture is dramatically reduced in the disadvantageous social comparison context, in which an individual is informed that the other participant is earning more monetary reward for performing the same task. These findings suggest that social comparison can affect the reward-driven capture of attention.
\end{abstract}

Keywords Social comparison · Involuntary attentional capture $\cdot$ Reward $\cdot$ Reward-driven attentional capture

It has been well documented that our spatial attention is guided by the physical salience of stimuli and by task goals (Corbetta \& Shulman, 2002; Du \& Abrams, 2012; Du, Zhang, \& Abrams, 2014). However, recent studies have shown that people tend to assign attentional priority to objects associated with rewards, even when they are task-irrelevant and nonsalient (Anderson, Laurent, \& Yantis, 2011; Della Libera \& Chelazzi, 2006; Hickey, Chelazzi, \& Theeuwes, 2010). For

J. Jiao $\cdot$ F. Du $(\bowtie) \cdot$ X. He $\cdot$ K. Zhang

Key Laboratory of Behavioral Science, Institute of Psychology,

Chinese Academy of Science, 16 Lincui Road,

Beijing, China 100101

e-mail:duf@psych.ac.cn

J. Jiao

University of Chinese Academy of Sciences, Beijing, China instance, Anderson et al. (2011) required participants to search for two pre-specified color targets (e.g., red and green), each of which was associated with either a high-value or a lowvalue monetary reward during training. In the subsequent test, participants were required to search for a shape singleton target without rewards, while reward-related colors were sometimes presented as distractors. Anderson et al. (2011) found that only the high-value reward-related color delayed response time to find the shape singleton target. Additionally, Anderson and Yantis (2013) found that the reward-related color captured attention when presented as an irrelevant distractor over half a year later. These findings indicate that rewards have a longlasting effect on involuntary capture of attention. Yet it is largely unknown whether and how high-level social manipulations (e.g., social comparison) affect involuntary attentional capture.

Several studies have shed new light on this issue by demonstrating that social comparison modulates reward processing (Fliessbach et al., 2007; Takahashi et al., 2009). For example, when two participants simultaneously performed a task with monetary reward in an fMRI study, Fliessbach et al. (2007) found that the magnitude of the neural responses in the ventral striatum was strongest when participants received more than their counterpart, intermediate for equal payments, and lowest when they received less than their counterpart. Moreover, people are usually dissatisfied with disadvantageous rewards and happy with advantageous rewards (Dvash et al., 2010; Qiu et al., 2010).

Although social comparison has been shown to modulate reward processing, few studies have examined whether social comparison can modulate involuntary attentional capture. Thus social comparison is particularly of interest in the present study. We speculated that social comparison could modulate reward-driven attentional capture. Specifically, an advantageous social context might magnify the subjective value of a reward, resulting in larger reward-driven attentional capture; 
in contrast, a disadvantageous social context might reduce the subjective value of a reward, leading to smaller reward-driven attentional capture.

Since Anderson et al.'s (2011) study bridges the areas of involuntary capture of attention and reward processing, the present study integrates social context manipulations with the value-driven attentional capture paradigm to examine whether social comparison can modulate attentional capture driven by a color distractor that has been previously associated with rewards. As in Anderson et al.'s (2011) study, the present study included training and test phases, but we added a social context in which participants were informed of not only their own rewards but also the rewards given to an imaginary partner in the training phase (see Fig. 1a). We set up four social comparison conditions (see Table 1) to examine their effects on reward-related capture of attention.

\section{Method}

\section{Participants}

Eighty naïve volunteers were randomly assigned to Experiments 1-4 (20 per experiment, aged 18-30 years; 39 males). There were nine participants for the control experiment (aged 20-28 years; four males), and another 20 for Experiment 5 (aged 19-26 years; 12 males). Each had normal or correctedto-normal vision and color vision.

Stimuli and procedure

In Experiments 1-4, stimuli were presented on a 17-in CRT with an $85 \mathrm{~Hz}$ refresh rate at a distance of $50 \mathrm{~cm}$. Each
Table 1 Manipulations of four social comparison conditions

\begin{tabular}{llll}
\hline Experiment & $\begin{array}{l}\text { Relative } \\
\text { reward level } \\
\text { (self:other) }\end{array}$ & $\begin{array}{l}\text { Absolute } \\
\text { reward } \\
\text { value }\end{array}$ & $\begin{array}{l}\text { Payoffs in Chinese } \\
\text { Yuan (cent) } \\
\text { (selfother) }\end{array}$ \\
\hline 1-Equivalent & $1: 1$ & High-Value & $10: 10$ \\
2-Disadvantageous & $1: 1$ & Low-Value & $12: 2$ \\
& $1: 2$ & High-Value & $10: 20$ \\
3-Advantageous & $1: 2$ & Low-Value & $12: 4$ \\
& $2: 1$ & High-Value & $10: 5$ \\
4-Inconsistent Ratio & $1: 1$ & Low-Value & $12: 1$ \\
& $2: 1$ & High-Value & $10: 20$ \\
& & Low-Value & $12: 1$
\end{tabular}

Note The table presents payoff conditions during the training phase when both participants and their imaginary partner were correct. When both participants and their imaginary partner responded incorrectly, neither received anything. When only one of the participants' responses were correct, he/she received some monetary reward while the other received no reward

experiment contained a training phase (Fig. 1a) and a test phase (Fig. 1b).

During training, a central fixation cross was presented for a period randomly chosen from 400, 500, or $600 \mathrm{~ms}$, followed by the search display which was presented until a response was made or $600 \mathrm{~ms}$ had elapsed. Participants had another 1, 000-ms interval to make a response if they did not respond within $600 \mathrm{~ms}$ of presentation of the search display. The search display consisted of six circles $\left(2.3^{\circ} \times 2.3^{\circ}\right)$, which were arranged equally in a circular array ( $5^{\circ}$ radius). Each shape contained a small, white line tilted $45^{\circ}$ to either the left or the right; only the line within the target was horizontal or vertical. The colors of the five distractor circles were

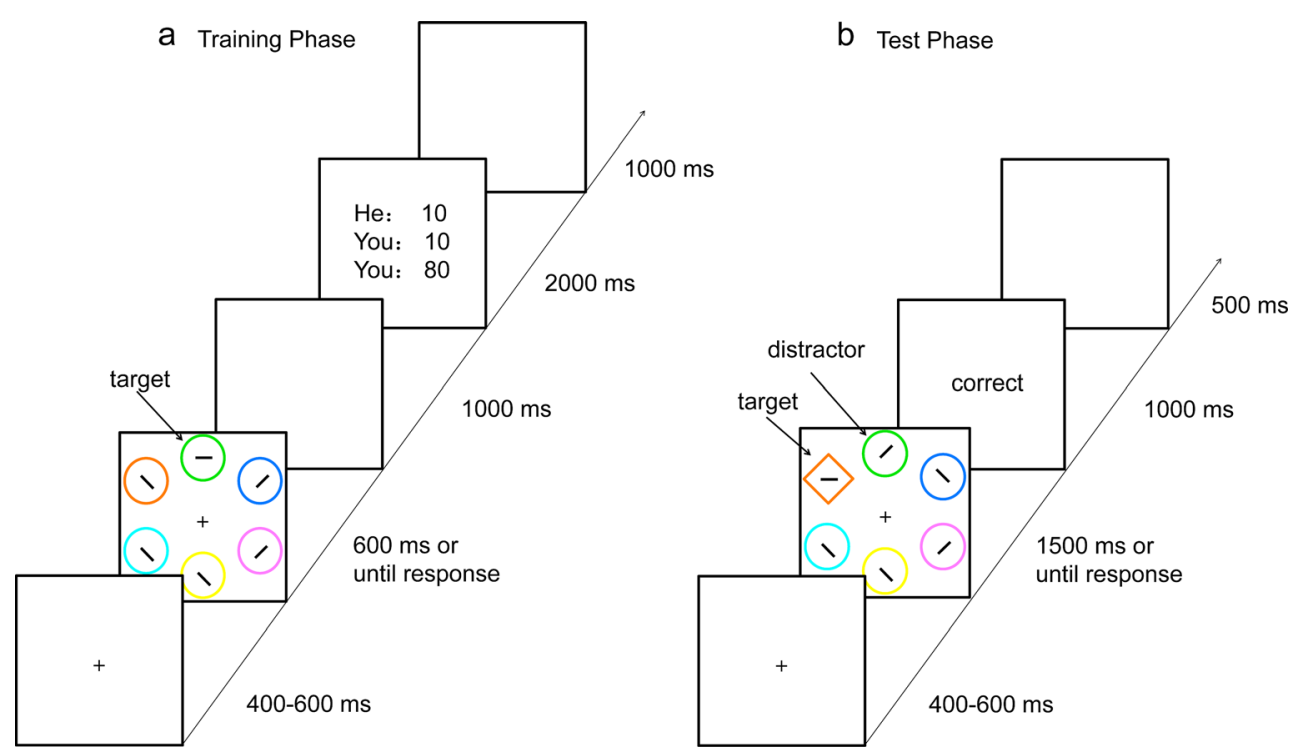

Fig. 1 The sequence of events in a typical trial. The graph in (a) illustrates events in the training phase and (b) illustrates events in the test phase 
randomly chosen from blue, cyan, pink, orange, yellow, and white. The target was either a red or green circle with equal probability. The participant received feedback $1,000 \mathrm{~ms}$ after a response about the reward value to himself/herself and the imaginary partner for that trial, together with their cumulative reward. The feedback lasted for $2,000 \mathrm{~ms}$. The feedback procedure was designed to provoke certain kinds of social comparison. The inter-trial interval was $1,000 \mathrm{~ms}$. Correct responses to high-value targets were rewarded with a highvalue (10 cents) on $80 \%$ of trials and a low value ( 2 cents) on the remaining $20 \%$. For low-value targets, the percentages were reversed. High-value targets were red for half of the participants, and green for the other half.

In the test phase, the search display consisted of six different colored shapes, which remained present until a response was made or 1,500 ms had elapsed. The target was defined as a shape singleton, which could be either a circle among five diamonds or a diamond among five circles. There were three distractor conditions: (1) the high-value distractor condition ( $25 \%$ of trials): one of the five non-target shapes was in the color associated with the high-value reward during training, (2) the low-value distractor condition ( $25 \%$ of trials): one of the five non-target shapes was in the color associated with the low-value reward; and (3) the neutral condition (50\% of trials): all five non-target shapes were in the color not associated with reward during training. The feedback was presented after response for $1,000 \mathrm{~ms}$. The inter-trial interval was $500 \mathrm{~ms}$.

Both the training and test phases consisted of 480 experimental trials. Participants performed 50 practice trials before the training phase and 20 practice trials before the test phase.

To encourage participants to make social comparisons, participants were told that another participant would perform the experiment simultaneously with them in the other laboratory room. However, they were clearly informed that their reward was independent of their partner's reward. Moreover, an independent sample of eight participants only took the training phase and showed an average correct response rate of $95 \%$ (89-98 \%). Thus the mean accuracy of the imaginary participant was set at $95 \%$ to make the experimental set-up more realistic. At the end of the experiment, all participants asked how much their partner earned, indicating that they all believed in the existence of the imaginary participant.

\section{Design}

As shown in Table 1, four different social comparison conditions were tested in Experiments 1-4: (1) The Equivalent reward condition (Self:Other $=1: 1$ ) was tested in Experiment 1, in which participants received an equal amount of reward to their imaginary partner; (2) The Disadvantageous reward condition (Self:Other $=1: 2$ ) was tested in Experiment 2. Whenever participants received a reward, their imaginary partner received a reward that was twice as valuable as they did; (3)
The Advantageous reward condition (Self:Other $=2: 1$ ) was tested in Experiment 3. Whenever participants received a reward, their imaginary partner received only half of the reward that they did; and (4) The Inconsistent Ratio reward condition was tested in Experiment 4. Whenever participants received a high-value reward, their imaginary partner received twice as much as they did (Self:Other =1:2); but when they received a low-value reward, their imaginary partner received only half of the reward that they did (Self:Other $=2: 1$ ).

Experiment 5 was a control experiment that had the same parameters as those in Experiment 2 (disadvantageous reward) with two exceptions. First, participants were only informed of their own reward during training so that they could not compare with others. Second, participants took an unrelated dots-estimation task with a higher reward (20 cents) before they performed the training and test phases. Thus, even without social comparison, the high-value reward in training was disadvantageous compared to rewards in the dotsestimation task.

In the unrelated dots-estimation task, a number of dots (1150) were first presented for $1,500 \mathrm{~ms}$. Participants were required to judge whether the number of dots was less or more than 24 within another 1,500 ms. A 20-cent reward was given for a correct response and no reward for an incorrect response. There were ten practice trials prior to 80 experimental trials. The mean accuracy was $93 \%$.

\section{Results}

As in Anderson et al. (2011), we used response times (RTs) in the test phase to measure the involuntary capture of attention. Mean RTs for correct trials and error rates were submitted to a $4 \times 3$ ANOVA with social comparison condition (equivalent, disadvantageous, advantageous, and inconsistent ratio) as a between-subjects factor and distractor condition (high-value, low-value, and neutral) as a within-subjects factor. Across all four experiments, error rates were generally low (see Table 2 ). There was no significant effect on error rates (all $F_{\mathrm{S}}<1.4$, all $p \mathrm{~s}>.25)$. However, the analysis of RTs revealed a significant main effect of distractor condition, $F(2,152)=39.334, p<$

Table 2 Mean error rates (in percentages) and standard errors (in parentheses) as a function of distractor condition in the test phase (for experiments 1-4)

\begin{tabular}{llll}
\hline Experiment & \multicolumn{3}{l}{ Distractor condition in the test phase } \\
\cline { 2 - 4 } & High-value & Low-value & Neutral \\
\hline 1-Equivalent & $8.3(1.1)$ & $7.9(1.0)$ & $7.6(0.8)$ \\
2-Disadvantageous & $7.3(1.2)$ & $6.4(0.8)$ & $6.9(1.0)$ \\
3-Advantageous & $7.6(0.7)$ & $7.8(1.0)$ & $7.9(0.9)$ \\
4-Inconsistent Ratio & $8.1(1.2)$ & $8.5(1.4)$ & $8.5(1.0)$ \\
\hline
\end{tabular}


$.001, \eta_{p}{ }^{2}=.341$, but the main effect of social comparison condition was not significant, $F(3,76)=1.106, p=.352$, $\eta_{p}{ }^{2}=.042$. Most importantly, the interaction was significant, $F(6,152)=4.234, p=.001, \eta_{p}{ }^{2}=.143$. Thus we performed one-way ANOVA with distractor condition as a within-subject factor for each experiment.

Finally, since the equivalent condition provided a baseline for reward-driven attentional capture, we also conducted a 2 (social comparison condition) $\times 3$ (distractor condition) ANOVA to compare the reward-driven attentional capture of the equivalent condition against the other three social comparison conditions (disadvantageous, advantageous, and inconsistent ratio).

\section{Experiment 1: equivalent reward}

As shown in Fig. 2a, Mean RTs differed significantly in the three distractor conditions, $F(2,38)=13.778, p<.001, \eta_{p}{ }^{2}=$ .420 , with RTs for the high-value distractor trials (749 ms) longer than RTs for the low-value distractor $(720 \mathrm{~ms})$ and neutral $(709 \mathrm{~ms})$ trials, both $p \mathrm{~s}<.01$ with Bonferroni correction. The difference between the latter two conditions was not significant, $p>.40$.

In addition, nine new participants took an additional control experiment including only the test phase. The result showed that a red distractor $(789 \mathrm{~ms})$, a green distractor $(791 \mathrm{~ms})$, or no color distractor $(790 \mathrm{~ms})$ produced
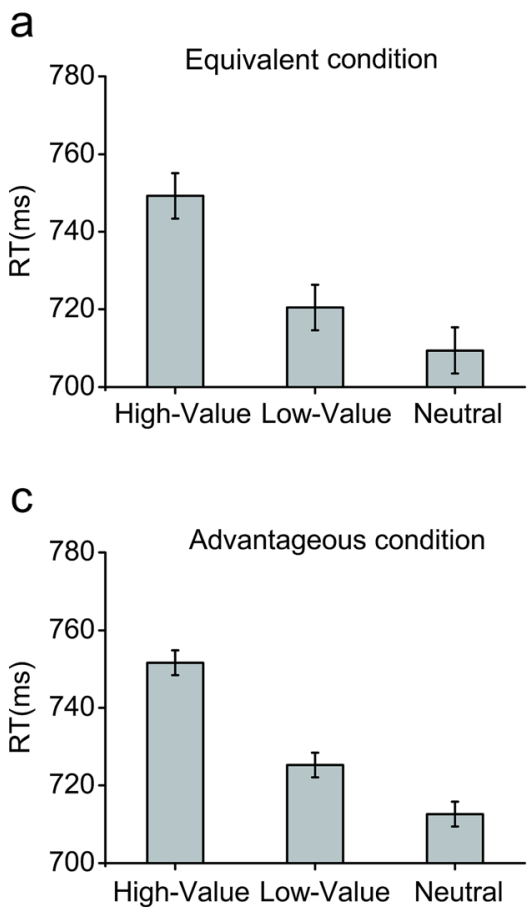

Fig. 2 Mean response times (RTs) by distractor condition during the test phase in (a) Experiment 1-Equivalent condition, (b) Experiment 2Disadvantageous condition, (c) Experiment 3-Advantageous condition, comparable RTs, $F<1$, indicating that red, green, and other colors were equally salient.

Experiment 1 replicated the primary result of Anderson et al.'s (2011) study - an irrelevant color distractor which was previously associated with a high-value reward can involuntarily capture attention, disrupting a subsequent search for a shape target.

Experiment 2: disadvantageous reward

Participants always received a disadvantageous reward compared with their imaginary partner irrespective of reward value. As illustrated in Fig. 2b, there was no significant effect of distractor condition, $F(2,38)=1.517, p=.232, \eta_{p}{ }^{2}=.074$, indicating comparable RTs for the high-value distractor (772 ms), low-value distractor $(769 \mathrm{~ms})$, and neutral (762 ms) trials.

To investigate whether a disadvantageous reward modulates the involuntary capture of a color distractor associated with a reward, RTs of Experiments 1 and 2 (equivalent and disadvantageous rewards) were submitted to a mixed-model ANOVA with experiment as a between-subjects factor and distractor condition as a within-subject factor. The difference between experiments was not significant, $F(1,38)=2.092, p$ $=.156, \eta_{p}{ }^{2}=.052$. However, there was an effect of distractor condition, $F(2,76)=13.236, p<.001, \eta_{p}{ }^{2}=.258$. Most importantly, the effect of distractor condition varied across

b

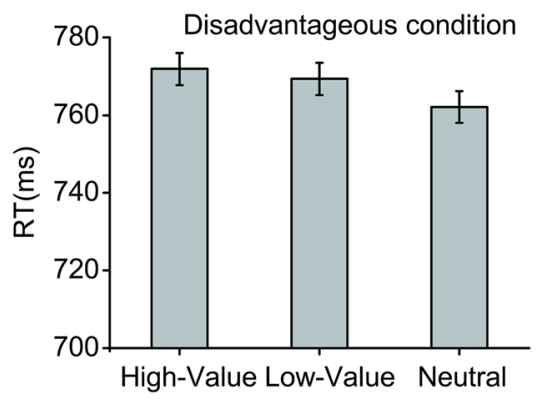

d

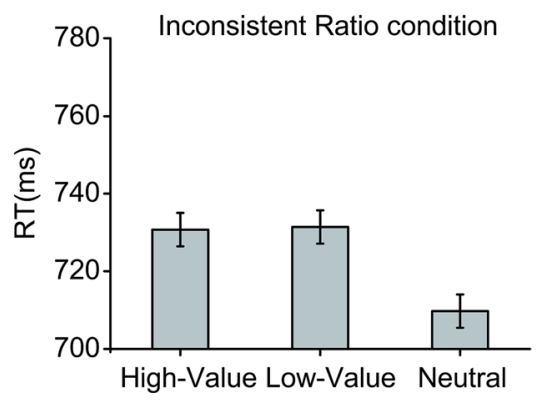

and (d) Experiment 4-Inconsistent Ratio condition. Error bars represent the $95 \%$ confidence intervals for the respective within-subject contrasts (Loftus \& Masson, 1994) 
the two experiments, $F(2,76)=5.641, p=.005, \eta_{p}{ }^{2}=.129$. Specifically, the high-value distractor significantly impaired a subsequent search for a form singleton target in Experiment 1 (equivalent reward) but not in Experiment 2 (disadvantageous reward). Moreover, we also compared the capture effect for the high-value distractor of the two experiments (the capture effect was estimated by subtracting the RTs in the neutral trials from the RTs in the high-value condition). The capture effect induced by the high-value distractor $(9.7 \mathrm{~ms})$ in Experiment 2 was significantly smaller than that in Experiment 1 ( $39.8 \mathrm{~ms})$, $t(38)=2.812, p<.01$. These results indicate that the disadvantageous reward context significantly reduced the attentional priority of a color distractor that was previously associated with the high-value rewards.

It is also possible that the reduced attentional priority of a high-value-reward-associated distractor was due to its short life-span of attentional priority. We further analyzed the RTs of the first half of the trails (240 trials) of the test phase. However, in this case, RTs for the high-value distractor (794 ms), low-value distractor (792 ms), and neutral $(783 \mathrm{~ms})$ trials were still not different, $F(2,38)=1.247, p=$ $.299, \eta_{p}{ }^{2}=.062$. Therefore, a high-reward-associated distractor does not have attentional priority at the first half of the test phase.

\section{Experiment 3: advantageous reward}

Participants were always given advantageous rewards compared with their imaginary partner irrespective of reward value. As shown in Fig. 2c, RTs differed significantly in the three distractor conditions, $F(2,38)=39.029, p<.001, \eta_{p}{ }^{2}=.673$. Both the high-value $(752 \mathrm{~ms})$ and low-value $(725 \mathrm{~ms})$ distractors slowed RT compared to the neutral condition (713 ms), both $p s<.05$. Furtermore, the high-value distractor delayed RT more than the low-value distractor, $p<.001$.

Comparison of RTs between Experiments 1 and 3 (equivalent and advantageous rewards) showed a main effect of distractor condition, $F(2,76)=39.930, p<.001, \eta_{p}{ }^{2}=$ .512 . However, there was neither a difference between experiments nor an interaction, both $F_{\mathbf{s}}<1$. Thus the advantageous reward condition mainly replicated the finding that the highvalue distractor can capture attention involuntarily.

We also carried out a comparison between Experiments 2 and 3 (disadvantageous and advantageous rewards). Again, there was a main effect of distractor condition, $F(2,76)=$ $22.267, p<.001, \eta_{p}{ }^{2}=.369$. However, there was no difference between experiments, $F(1,38)=1.872, p=.179, \eta_{p}{ }^{2}=.047$. Again, there was an interaction, $F(2,76)=8.812, p<.001$, $\eta_{p}{ }^{2}=.188$, indicating the effect of distractor condition varied significantly between the two experiments. The capture effect induced by the high-value distractor $(9.7 \mathrm{~ms})$ in Experiment 2 was significantly smaller than that in Experiment $3(38.9 \mathrm{~ms})$, $t(38)=3.996, p<.001$. Thus, the disadvantageous condition dramatically reduced the reward-driven attentional capture that was observed in the advantageous condition.

Experiment 4: inconsistent ratio reward

The results of Experiment 2 (disadvantageous) and Experiment 3 (advantageous conditions) showed that the disadvantageous condition substantially reduced the attentional priority of a color distractor that was previously associated with the high-value rewards, while the advantageous social comparison only numerically enhanced the priority of distractors previously associated with low-value rewards. We designed Experiment 4 to further examine these findings, in which participants were disadvantaged compared to their imaginary partner for high-value rewards, but were advantageous for lowvalue rewards. Thus we expected reduced capture by the highvalue distractors and possibly slightly enhanced capture by the low-value distractors.

As shown in Fig. 2d, there was a significant effect of distractor condition, $F(2,38)=8.095, p<.001, \eta_{p}{ }^{2}=.299$. Specifically, RTs in the high-value distractor condition (731 ms) and the low-value distractor condition $(731 \mathrm{~ms})$ were significantly longer than those in the neutral condition (710 ms), both $p \mathrm{~s}<.05$. The comparison between Experiments 1 and 4 (equivalent and inconsistent ratio rewards) revealed a main effect of distractor condition, $F(2,76)=$ 18.739, $p<.001, \eta_{p}{ }^{2}=.330$, and an interaction, $F(2,76)=$ $4.525, p=.014, \eta_{p}{ }^{2}=.106$. Again there was no main effect of experiment, $F<1$. The capture effect induced by the highvalue distractor $(20.9 \mathrm{~ms})$ in Experiment 4 was slightly smaller than that in Experiment 1 (39.8 ms), $t(38)=2.244, p<.05$, one-tailed. However, there was no significant difference between the magnitude of capture induced by the low-value distractor $(21.6 \mathrm{~ms})$ in Experiment 4 and that induced by the similar distractor in Experiment 1 (11 ms), $p>.05$.

As expected, we again found that the disadvantageous social comparison reduced attentional priority of a high-valuereward-associated distractor, but the advantageous social comparison did not significantly enhance the priority of distractors associated with low-value rewards.

\section{Experiment 5: higher reward context}

Though the disadvantageous reward consistently reduced the attentional priority of previously rewarded distractors, the modulation could be driven by reward context instead of social comparison. For example, the fact that participants observe a 20 -cent reward being granted in the disadvantageous reward might desensitize participants to the size of their own rewards and make the 10-cent reward no longer a "high" reward.

To test this possibility, we conducted Experiment 5 which provided a higher reward context as the disadvantageous 
context, but without social comparison. We found a significant effect of distractor condition, $F(2,38)=13.207, p<.001, \eta_{p}{ }^{2}$ $=.410$, with RTs for the high-value distractor trials $(772 \mathrm{~ms})$ longer than RTs for the neutral trials (742 ms), $p=.001$. RTs for the low-value distractor $(755 \mathrm{~ms})$ trials were only numerically longer than the neutral trials, $p=.134$.

The comparison of RTs between Experiments 2 and 5 (disadvantageous and higher rewards) showed a main effect of distractor condition, $F(2,76)=11.664, p<.001, \eta_{p}{ }^{2}=.235$. However, the difference between experiments was not significant, $F(1,38)=.143, p=.708, \eta_{p}^{2}=.004$. Crucially, the effect of distractor condition varied across experiments, $F(2$, 76) $=3.151, p<.05, \eta_{p}{ }^{2}=.077$. The capture effect induced by the high-value distractor $(9.7 \mathrm{~ms})$ in Experiment 2 was significantly smaller than that in Experiment $5(30 \mathrm{~ms}), t(38)=$ $2.273, p=.029$. Thus, the reduced reward-driven capture effect in the disadvantageous social context could not be explained by reward context per se.

\section{Discussion}

The present study found that reward-driven attentional capture was dramatically reduced in the disadvantageous social context. Moreover, the absence of value-driven capture in the disadvantageous social context cannot be explained by the higher reward context which only provides comparison between two values without social comparison. Nevertheless, advantageous social comparison seems insufficient to magnify reward-driven attentional capture. The present study is the first study to demonstrate that social comparison can modulate involuntary reward-related attentional capture.

Several underlying mechanisms might contribute to this modulatory effect. First, social comparison can modulate reward processing (Fliessbach et al., 2007; Takahashi et al., 2009), which in turn might either accelerate or hinder perceptual learning of attentional bias to reward-associated stimuli (Anderson et al., 2011). The disadvantageous social context might indirectly impair perceptual learning by reducing the subjective value of rewards, thus resulting in less valuedriven capture.

Alternatively, ample evidence shows that different emotional responses might be evoked by different social contexts accompanying the reward processing (Dvash et al., 2010; Takahashi et al., 2009). A disadvantageous context is likely to evoke envy and dissatisfaction, while an advantageous context will induce "schadenfreude." As emotion also plays an important role in learning and memory (D'Mello, Lehman, Pekrun, \& Graesser, 2014), these emotional responses, especially dissatisfaction evoked by social comparisons, might have impeded perceptual learning of reward-related stimuli during the training, resulting in a weaker association between rewards and stimuli.
Finally, it is worth noting that the neural networks for social comparison and selective attention partially overlap. Several functional magnetic resonance imaging studies have shown that various brain regions, such as the ventral striatum (Fliessbach et al., 2007), dorsolateral prefrontal cortex (DLPFC), and anterior cingulate cortex (ACC) (Takahashi et al., 2009) are involved in social comparison. It is well known that both the ACC and the DLPFC are also important nodes of the attentional network (Corbetta \& Shulman, 2002). The partial overlap between these two neural networks might also imply that social comparison may modulate allocation of attention in training. The altered attentional priority of those color stimuli were then transferred to a new task. These possibilities require further examination in future research.

A wide variety of rewards including food, nicotine, emotionally provoking pictures, money, and drugs can substantially shape voluntary behavior. By associative learning, the recipient of a rewarding stimulus might have a desire or "wanting" response when reward-associated stimuli are present (Robinson \& Berridge, 2008), resulting in involuntary capture of a rewarding stimulus. Such involuntary capture by cues associated with rewarding stimuli has been reported among smokers and substance abusers (Field, Mogg, \& Bradley, 2004). The present findings might provide a novel way to reduce the possibility that the attention of addicted individuals would be captured by reward-related cues. It is definitely worth further examination whether a disadvantageous social comparison context can reduce or even completely eliminate the capturing effect of reward-related cues.

In summary, the present results provide evidence that a disadvantageous social comparison context remarkably reduces reward-driven attentional capture.

Acknowledgments This study was supported by the National Natural Science Foundation of China (31470982 and 31200766), the Chinese Academy of Sciences (grant number KJZD-EW-L04), and the scientific foundation of the Institute of Psychology, Chinese Academy of Sciences (Y4CX033008). We thank two anonymous reviewers for their valuable comments and suggestions on earlier versions of this article, and Richard Carciofo for proofreading this article.

\section{References}

Anderson, B. A., Laurent, P. A., \& Yantis, S. (2011). Value-driven attentional capture. Proceedings of the National Academy of Sciences of the United States of America, 108, 10367-10371. doi:10.1073/pnas. 1104047108

Anderson, B. A., \& Yantis, S. (2013). Persistence of value-driven attentional capture. Journal of Experimental Psychology: Human Perception and Performance, 39, 6-9.

Corbetta, M., \& Shulman, G. L. (2002). Control of goal-directed and stimulus-driven attention in the brain. Nature Reviews Neuroscience, 3, 201-215. 
D’Mello, S., Lehman, B., Pekrun, R., \& Graesser, A. (2014). Confusion can be beneficial for learning. Learning and Instruction, 29, 153170

Della Libera, C., \& Chelazzi, L. (2006). Visual selective attention and the effects of monetary rewards. Psychological Science, 17, 222-227. doi:10.1111/j.1467-9280.2006.01689.x

Du, F., \& Abrams, R. A. (2012). Out of control: Attentional selection for orientation is thwarted by properties of the underlying neural mechanisms. Cognition, 124, 361-366.

Du, F., Zhang, K., \& Abrams, R. A. (2014). Hold the future, let the past go: Attention prefers the features of future targets. Cognition, 131, 205-215.

Dvash, J., Gilam, G., Ben-Ze'ev, A., Hendler, T., \& Shamay-Tsoory, S. G. (2010). The envious brain: The neural basis of social comparison. Human Brain Mapping, 31, 1741-1750.

Field, M., Mogg, K., \& Bradley, B. P. (2004). Eye movements to smoking-related cues: effects of nicotine deprivation. Psychopharmacology, 173, 116-123.

Fliessbach, K., Weber, B., Trautner, P., Dohmen, T., Sunde, U., Elger, C. E., \& Falk, A. (2007). Social comparison affects reward-related brain activity in the human ventral striatum. Science, 318, 1305-1308. doi:10.1126/science.1145876

Hickey, C., Chelazzi, L., \& Theeuwes, J. (2010). Reward changes salience in human vision via the anterior cingulate. Journal of Neuroscience, 30, 11096-11103.

Loftus, G. R., \& Masson, M. (1994). Using confidence intervalsin within-subject designs. Psychonomic Bulletin \& Review, 4, 476-490.

Qiu, J., Yu, C. Y., Li, H., Jou, J. W., Tu, S., Wang, T., Wei, D. T., Zhang, Q. L. (2010). The impact of social comparison on the neural substrates of reward processing: An event-related potential study. NeuroImage, 49(1), 956-962.

Robinson, T. E., \& Berridge, K. C. (2008). The incentive sensitization theory of addiction: some current issues. Philosophical Transactions of the Royal Society B-Biological Sciences, 363, 3137-3146. doi:10. 1098/rstb.2008.0093

Takahashi, H., Kato, M., Matsuura, M., Mobbs, D., Suhara, T., \& Okubo, Y. (2009). When Your Gain Is My Pain and Your Pain Is My Gain: Neural Correlates of Envy and Schadenfreude. Science, 323, $937-$ 939. doi:10.1126/science.1165604 